\title{
Optimal Verification of Greenberger-Horne-Zeilinger States
}

\author{
Zihao Li, ${ }^{1,2}$ Yun-Guang Han, ${ }^{1,2}$ and Huangjun Zhu ${ }^{1,2,3,4, \text { * }}$ \\ ${ }^{1}$ Department of Physics and Center for Field Theory and Particle Physics, Fudan University, Shanghai 200433, China \\ ${ }^{2}$ State Key Laboratory of Surface Physics, Fudan University, Shanghai 200433, China \\ ${ }^{3}$ Institute for Nanoelectronic Devices and Quantum Computing, Fudan University, Shanghai 200433, China \\ ${ }^{4}$ Collaborative Innovation Center of Advanced Microstructures, Nanjing 210093, China
}

(Dated: May 19, 2020)

\begin{abstract}
We construct optimal protocols for verifying qubit and qudit GHZ states using local projective measurements. When the local dimension is a prime, an optimal protocol is constructed from Pauli measurements only. Our protocols provide a highly efficient way for estimating the fidelity and certifying genuine multipartite entanglement. In particular, they enable the certification of genuine multipartite entanglement using only one test when the local dimension is sufficiently large. By virtue of adaptive local projective measurements, we then construct protocols for verifying GHZlike states that are optimal over all protocols based on one-way communication. The efficiency can be improved further if additional communications are allowed. Finally, we construct optimal protocols for verifying GHZ states and nearly optimal protocols for GHZ-like states in the adversarial scenario.
\end{abstract}

\section{INTRODUCTION}

Greenberger-Horne-Zeilinger (GHZ) states [1, 2] are typical examples of quantum states with genuine multipartite entanglement (GME) [3]. They play key roles both in quantum information processing and in foundational studies, such as quantum secret sharing 4, 5, entanglement purification [6], open-destination teleportation [7, quantum networks [8, randomness verification [9, and multipartite nonlocality tests [10, 11]. The significance of GHZ states is witnessed by numerous experiments devoted to preparing them in various platforms, with ever-increasing number of particles [11 18]. In practice, multipartite quantum states prepared in experiments are never perfect, so it is crucial to verify these states with high precision using limited resources. However, traditional tomographic approaches are known to be resource consuming and very inefficient [12, 17, 19]. Even with popular alternatives like direct fidelity estimation [20, the scaling behaviors of the number of required measurements with the infidelity and the qubit number are suboptimal.

Recently, an alternative approach known as quantum state verification has attracted increasing attention [2126. Efficient verification protocols based on local operations and classical communication (LOCC) have been constructed for stabilizer states [24, 26, 29, hypergraph states 29], and Dicke states [30. However, optimal protocols are known only for maximally entangled states [21, 31, 32] and bipartite pure states under restricted LOCC [24, 33 35]. For quantum states with GME, such as GHZ states, no optimal protocol has been found so far because such optimization problems are usually extremely difficult. In addition, most protocols known so far are not homogeneous, which is not desirable for practical applications [26. Any progress on these issues is of

\footnotetext{
* zhuhuangjun@fudan.edu.cn
}

interest to both theoretical studies and practical applications.

In this paper, we propose optimal protocols for verifying (qubit and qudit) GHZ states using local projective measurements. When the local dimension is a prime, only Pauli measurements are required. Moreover, all the protocols we construct are homogeneous. They offer a highly efficient tool for fidelity estimation and entanglement certification. Surprisingly, the GME can be certified with any given significance level using only one test when the local dimension is sufficiently large, which has never been achieved or even anticipated before. By virtue of adaptive local projective measurements, our protocols can be generalized to GHZ-like states, while retaining the high efficiency. Moreover, these protocols can be applied to the adversarial scenario with minor modification. Now the protocols for verifying GHZ states based on local projective measurements are actually optimal among all possible protocols without locality restriction. Besides quantum state verification, our protocols are also useful for verifying quantum gates, including some Clifford gates and the controlled-swap (CSWAP) gate [36].

\section{PURE-STATE VERIFICATION}

\section{A. Basic framework}

Before proposing protocols for verifying GHZ states, let us briefly review the general framework of pure-state verification 24 26]. Consider a quantum device that is supposed to produce the target state $|\Psi\rangle \in \mathcal{H}$, but actually produces the states $\sigma_{1}, \sigma_{2}, \ldots, \sigma_{N}$ in $N$ runs. Our task is to verify whether these states are sufficiently close to the target state on average. To achieve this task, we can perform two-outcome measurements $\left\{E_{l}, \mathbb{1}-E_{l}\right\}$ from a set of accessible measurements (projective measurements are most appealing in practice, but our discussions apply to general measurements). Each mea- 
surement represents a test, and the test operator $E_{l}$ corresponds to passing the test. Here we require that the target state $|\Psi\rangle$ can always pass the test, that is, $E_{l}|\Psi\rangle=|\Psi\rangle$. Suppose the test $\left\{E_{l}, \mathbb{1}-E_{l}\right\}$ is performed with probability $p_{l}$, then the verification operator (also called a strategy) is given by $\Omega=\sum_{l} p_{l} E_{l}$. If $\left\langle\Psi\left|\sigma_{j}\right| \Psi\right\rangle \leq 1-\varepsilon$, then the average probability that $\sigma_{j}$ can pass each test satisfies [24, 26]

$$
\operatorname{tr}\left(\Omega \sigma_{j}\right) \leq 1-[1-\beta(\Omega)] \varepsilon=1-\nu(\Omega) \varepsilon,
$$

where $\beta(\Omega)$ denotes the second largest eigenvalue of $\Omega$, and $\nu(\Omega):=1-\beta(\Omega)$ is the spectral gap from the maximum eigenvalue. The inequality in Eq. (1) is saturated when $\left\langle\Psi\left|\sigma_{j}\right| \Psi\right\rangle=1-\varepsilon$ and $\sigma_{j}$ is supported on the subspace associated with the two largest eigenvalues of $\Omega$.

Suppose the states $\sigma_{1}, \sigma_{2}, \ldots, \sigma_{N}$ are independent of each other and let $\varepsilon_{j}=1-\left\langle\Psi\left|\sigma_{j}\right| \Psi\right\rangle$. Then the probability that these states can pass all $N$ tests satisfies the following tight upper bound [25, 26]

$$
\prod_{j=1}^{N} \operatorname{tr}\left(\Omega \sigma_{j}\right) \leq \prod_{j=1}^{N}\left[1-\nu(\Omega) \varepsilon_{j}\right] \leq[1-\nu(\Omega) \bar{\varepsilon}]^{N},
$$

where $\bar{\varepsilon}=\sum_{j} \varepsilon_{j} / N$ is the average infidelity. In order to ensure the condition $\bar{\varepsilon}<\varepsilon$ with significance level $\delta$, that is, to ensure the condition $\prod_{j} \operatorname{tr}\left(\Omega \sigma_{j}\right) \leq \delta$ when $\bar{\varepsilon} \geq \varepsilon$, it suffices to perform [25, 26]

$$
N=\left\lceil\frac{\ln \delta}{\ln [1-\nu(\Omega) \varepsilon]}\right\rceil \approx \frac{\ln \delta^{-1}}{\nu(\Omega) \varepsilon}
$$

tests. To minimize the number of tests, we need to maximize the value of the spectral gap $\nu(\Omega)$ under LOCC. This task is usually extremely difficult if not impossible. It should be pointed out that the approximation in Eq. (3) is valid only when $\nu(\Omega) \varepsilon \ll 1$. Otherwise, the minimum number of tests required is more sensitive to the spectral gap $\nu(\Omega)$, which has an important implication for entanglement certification, as we shall see in Sec. IIID,

In the above presentation we follow the assumptions and interpretation in Refs. 25, 26, in contrast to the counterpart in Ref. 24. The authors in Ref. 24 assume that either $\sigma_{j}=|\Psi\rangle\langle\Psi|$ for all $j$ (good case) or $\varepsilon_{j} \geq \varepsilon$ for all $j$ (bad case), and the task is to distinguish which case occurs. However, this assumption is difficult to guarantee and is not feasible from a practical point of view. The assumptions in Refs. [25, 26] are more reasonable and the conclusion is stronger in comparison. In particular, the average fidelity rather than the maximum fidelity of the prepared states $\sigma_{1}, \sigma_{2}, \ldots, \sigma_{N}$ can be verified.

\section{B. Homogeneous strategies}

A verification strategy is homogeneous if the verification operator $\Omega$ has the following form

$$
\Omega=|\Psi\rangle\langle\Psi|+\beta(\Omega)(\mathbb{1}-|\Psi\rangle\langle\Psi|)
$$

for some $0 \leq \beta(\Omega)<1$. Homogeneous strategies are most appealing for quantum state verification because of several important merits not shared by inhomogeneous strategies. To see this, let us consider a simple scenario in which all $\sigma_{1}, \sigma_{2}, \ldots, \sigma_{N}$ are identical to the state $\sigma$ with fidelity $\langle\Psi|\sigma| \Psi\rangle=F=1-\varepsilon$. If $\Omega$ is homogeneous, then the bounds in Eqs. (1) and (2) are saturated, so there is a simple connection between the passing probability and the fidelity of the states prepared, namely, $\operatorname{tr}(\sigma \Omega)=$ $[1-\beta(\Omega)] F+\beta(\Omega)$, which implies that

$$
F=\frac{\operatorname{tr}(\Omega \sigma)-\beta(\Omega)}{\nu(\Omega)}, \quad 1-F=\frac{1-\operatorname{tr}(\Omega \sigma)}{\nu(\Omega)}
$$

Based on this connection, we can estimate the fidelity and infidelity accurately given sufficiently many tests. According to Ref. [26], the standard deviation of this estimation is

$$
\Delta F=\frac{\sqrt{(1-F)\left(F+\nu^{-1}-1\right)}}{\sqrt{N}} \leq \frac{1}{2 \nu \sqrt{N}},
$$

where $\nu=\nu(\Omega)=1-\beta(\Omega)$ and $N$ is the number of tests performed.

If the strategy $\Omega$ is inhomogeneous by contrast, given the passing probability we can only derive lower and upper bounds for the infidelity [26]

$$
\frac{1-\operatorname{tr}(\Omega \sigma)}{1-\tau(\Omega)} \leq 1-F \leq \frac{1-\operatorname{tr}(\Omega \sigma)}{\nu(\Omega)}
$$

where $\tau(\Omega)$ is the smallest eigenvalue of $\Omega$. The lower bound in Eq. (7) is saturated when $\sigma$ is supported on the subspace associated with the largest and the smallest eigenvalues of $\Omega$. When the verification operator $\Omega$ is singular, that is, $\tau(\Omega)=0$, the upper bound is $1 / \nu(\Omega)$ times as large as the lower bound. When $\nu(\Omega)=0.1$ for example, in the worst case we can only conclude (with a given significance level) that the infidelity is smaller than $0.1(0.5)$ even if the actual infidelity is only 0.01 (0.05). Such a conclusion is far from being satisfactory even though it is correct. When the verification protocol is applied to entanglement detection, this problem makes it much more difficult to detect entanglement. Unfortunately, the problem cannot be resolved by increasing the number of tests. Therefore, it is desirable to construct a homogeneous verification strategy whenever possible.

In addition, homogeneous strategies are appealing for quantum state verification in the adversarial scenario [25, 26. In particular, a homogeneous strategy is the most efficient among all verification strategies with the same spectral gap; it can achieve a much better scaling behavior in the number of tests compared with a singular strategy. 


\section{OPTIMAL VERIFICATION OF GHZ STATES}

Here we are mainly interested in GHZ states of the form [1, 2]

$$
\left|\mathrm{G}_{n}^{d}\right\rangle=\frac{1}{\sqrt{d}} \sum_{j=0}^{d-1}|j\rangle^{\otimes n}
$$

Previously, a coloring protocol was proposed in Ref. 29] (cf. Ref. 9]), which can achieve a spectral gap of $1 / 2$ using two settings based on Pauli measurements, but this protocol is not homogeneous (see TableI), and the verification operator is singular. For a bipartite maximally entangled state of the same local dimension, the maximum spectral gap of any verification operator based on LOCC (or separable measurements) is $d /(d+1)$ [21, 24, 31, 32. Obviously, the counterpart for GHZ states cannot be larger. Here we shall show that this upper bound can be saturated.

\section{A. Optimal verification of the $n$-qubit GHZ state}

First, we consider optimal verification of the $n$-qubit GHZ state $\left|G_{n}^{2}\right\rangle$ based on Pauli measurements. Recall that the Pauli group for each qubit is generated by three Pauli matrices,

$$
X=\left(\begin{array}{ll}
0 & 1 \\
1 & 0
\end{array}\right), \quad Y=\left(\begin{array}{cc}
0 & -\mathrm{i} \\
\mathrm{i} & 0
\end{array}\right), \quad Z=\left(\begin{array}{cc}
1 & 0 \\
0 & -1
\end{array}\right) .
$$

Denote by $I$ the identity operator on the Hilbert space of one party, then a Pauli measurement is specified by a string in $\{I, X, Y, Z\}^{n}$, which determines the Pauli operators measured on individual qubits; the identity means no measurement. The weight of the Pauli measurement is the number of terms in the string that are not equal to the identity. The Pauli measurement is complete if the weight is equal to $n$, that is, the string does not contain the identity. A test operator $E$ (and the corresponding test) based on a Pauli measurement is not admissible if there exists another test operator $E^{\prime}$ based on the same or a different Pauli measurement such that $E^{\prime} \leq E$ and $\operatorname{tr}\left(E^{\prime}\right)<\operatorname{tr}(E)$; otherwise, the test operator $E$ (and the corresponding test) is admissible. A Pauli measurement is admissible if at least one admissible test operator can be constructed from this Pauli measurement and not admissible otherwise.

Given a Pauli measurement, let $\left\{\Pi_{1}, \Pi_{2}, \ldots, \Pi_{q}\right\}$ be the set of projectors corresponding to the measurement outcomes. The canonical test projector is defined as

$$
P=\sum_{\left\langle\mathrm{G}_{n}^{2}\left|\Pi_{o}\right| \mathrm{G}_{n}^{2}\right\rangle>0} \Pi_{o}
$$

To guarantee that the target state $\left|\mathrm{G}_{n}^{2}\right\rangle$ can always pass the test, any other test operator $E$ satisfies $E \geq P$ and thus cannot be admissible. The Pauli measurement is admissible iff the canonical test projector is admissible. These observations reveal the crucial role of canonical test projectors in constructing an efficient verification protocol.

For example, the canonical test projector associated with $Z^{n}$ (understood as a Pauli string with $n$ Pauli operators equal to $Z$ ) reads

$$
P_{0}=(|0\rangle\langle 0|)^{\otimes n}+(|1\rangle\langle 1|)^{\otimes n}
$$

the test is passed iff the outcomes of all $Z$ measurements on individual qubits coincide. Given a string in $\{X, Y\}^{n}$, let $\mathscr{Y}$ be the set of parties that perform $Y$ measurements, then $\overline{\mathscr{Y}}:=\{1,2, \ldots, n\} \backslash \mathscr{Y}$ is the set of parties that perform $X$ measurements. When $|\mathscr{Y}|=2 t$ is even, the canonical test projector reads

$$
P_{\mathscr{Y}}=\frac{1}{2}\left[\mathbb{1}+(-1)^{t} \prod_{k \in \mathscr{Y}} Y_{k} \prod_{k^{\prime} \in \overline{\mathscr{Y}}} X_{k^{\prime}}\right]
$$

the test is passed iff the total number of outcomes -1 (either from $X$ or $Y$ measurements) has the same parity as $t$. The following lemma clarifies all admissible Pauli measurements and test operators for $\left|\mathrm{G}_{n}^{2}\right\rangle$; see Appendix A for a proof.

Lemma 1. The GHZ state $\left|\mathrm{G}_{n}^{2}\right\rangle$ has $1+2^{n-1}$ admissible Pauli measurements, namely $Z^{n}$ and all strings in $\{X, Y\}^{n}$ with even numbers of $Y$. The corresponding $1+2^{n-1}$ canonical test projectors in Eqs. 11 and 12 are the only admissible test operators.

Our verification protocol is composed of $1+2^{n-1}$ admissible tests in which the test $P_{0}$ is performed with probability $1 / 3$ and the other $2^{n-1}$ tests are performed with probability $1 /\left(3 \times 2^{n-2}\right)$ each. The verification operator reads

$$
\Omega_{\mathrm{I}}:=\frac{1}{3}\left(P_{0}+\frac{1}{2^{n-2}} \sum_{\mathscr{Y}} P_{\mathscr{Y}}\right)=\frac{1}{3}\left(\mathbb{1}+2\left|\mathrm{G}_{n}^{2}\right\rangle\left\langle\mathrm{G}_{n}^{2}\right|\right),
$$

which is homogeneous. Here the second equality is proved in Appendix $\mathrm{B}$. We have $\beta\left(\Omega_{\mathrm{I}}\right)=1 / 3$, and

$$
\nu\left(\Omega_{\mathrm{I}}\right)=\frac{2}{3}, \quad N\left(\Omega_{\mathrm{I}}\right) \approx \frac{3}{2 \varepsilon} \ln \delta^{-1} .
$$

This protocol is optimal among all protocols based on LOCC or separable measurements. Compared with the strategy in Ref. [24] which achieves $\nu=2^{n-1} /\left(2^{n}-1\right)$ with $2^{n}-1$ measurement settings, our strategy not only has a higher efficiency, but also requires fewer measurement settings, as illustrated in Table I. The protocol proposed in Ref. 29] requires much fewer measurement settings, but it is not homogeneous and thus has a number of drawbacks as mentioned in Sec. II B. The current protocol is the most appealing if it is not difficult to switch Pauli measurements, which is the case in most scenarios of practical interest. 
TABLE I. Comparison of verification strategies for the $n$-qudit GHZ state $\left|\mathrm{G}_{n}^{d}\right\rangle$ in Eq. 8 . Here $R_{d}(\Omega)$ denotes the range of the local dimension over which each strategy is applicable, $\nu(\Omega)$ denotes the spectral gap of each strategy, $N(\varepsilon, \delta, \Omega)$ denotes the number of tests required to verify the target state within infidelity $\varepsilon$ and significance level $\delta$, and $N_{\mathrm{MS}}(\Omega)$ denotes the number of potential measurement settings. Strategies $\Omega_{\mathrm{PLM}}$ and $\Omega_{\mathrm{ZH}}$ are proposed in Refs. 24] and [29], respectively; the other three strategies are proposed in this paper.

\begin{tabular}{c|c|c|c|c|c}
\hline \hline Strategy & $R_{d}(\Omega)$ & $\nu(\Omega)$ & Is $\Omega$ homogeneous? & $N(\varepsilon, \delta, \Omega)$ & $N_{\mathrm{MS}}(\Omega)$ \\
\hline$\Omega_{\mathrm{PLM}}[24]$ & $d=2$ & $2^{n-1} /\left(2^{n}-1\right)$ & Yes & $\left(2^{n}-1\right) 2^{1-n} \varepsilon^{-1} \ln \delta^{-1}$ & $2^{n}-1$ \\
$\Omega_{\mathrm{ZH}}[29]$ & $d \geq 2$ & $1 / 2$ & No & $2 \varepsilon^{-1} \ln \delta^{-1}$ & 2 \\
$\Omega_{\mathrm{I}}$ & $d=2$ & $2 / 3$ & Yes & $(3 / 2) \varepsilon^{-1} \ln \delta^{-1}$ & $2^{n-1}+1$ \\
$\Omega_{\mathrm{II}}$ & $d$ is odd prime & $d /(d+1)$ & Yes & $(d+1) d^{-1} \varepsilon^{-1} \ln \delta^{-1}$ & $d^{n-1}+1$ \\
$\Omega_{\mathrm{III}}$ & $d \geq 3$ & $d /(d+1)$ & Yes & $(d+1) d^{-1} \varepsilon^{-1} \ln \delta^{-1}$ & $\left\lceil\frac{3}{4}(d-1)^{2}\right\rceil^{n-1}+1$ \\
\hline \hline
\end{tabular}

Moreover, our protocol proposed above is essentially the unique optimal protocol based on Pauli measurements as shown in Proposition 1 below and proved in Appendix A. In particular, the number $1+2^{n-1}$ of (potential) measurement settings cannot be reduced. In addition, all canonical test projectors are required to construct a homogeneous strategy. It should be pointed out that there is some freedom in choosing the Pauli group: different choices are related to each other by local unitary transformations. Here we focus on the canonical Pauli group generated by Pauli matrices in Eq. (9) for each qubit; only nonadaptive Pauli measurements associated with this Pauli group are considered. Nevertheless, the test operators are not required to be projectors, although it turns out that this relaxation does not provide any advantage.

Proposition 1. Suppose $\Omega$ is an optimal verification strategy with $\nu(\Omega)=2 / 3$ for $\left|\mathrm{G}_{n}^{2}\right\rangle$ that is based on Pauli measurements. Then $\Omega=\Omega_{\mathrm{I}}$; in addition, $\Omega$ is composed of admissible tests with the same probabilities as in $\Omega_{\mathrm{I}}$.

Besides quantum state verification, our protocol is also useful for verifying quantum gates, including Clifford gates and the CSWAP gate. The basic idea of quantum gate verification is to feed some pure test states into the quantum gate or gate set to be verified, and then verify the output states 36 38. Our protocol is useful whenever some output states are equivalent to GHZ states under local Clifford transformations.

\section{B. Optimal verification of the $n$-qudit GHZ state}

Next, we generalize the above results to the qudit case, assuming that the local dimension $d$ is an odd prime. The qudit Pauli group is generated by the phase operator $Z$ and the shift operator $X$ defined as follows,

$$
Z|j\rangle=\omega^{j}|j\rangle, \quad X|j\rangle=|j+1\rangle, \quad \omega=\mathrm{e}^{2 \pi \mathrm{i} / d},
$$

where $j \in \mathbb{Z}_{d}$ and $\mathbb{Z}_{d}$ is the ring of integers modulo $d$.

The concepts of admissible Pauli measurements/test operators and canonical test projectors can be defined in a similar way as in Sec. IIIA One admissible test is associated with the Pauli measurement $Z^{n}$ with the canonical test projector

$$
P_{0}=\sum_{j=0}^{d-1}(|j\rangle\langle j|)^{\otimes n}
$$

the test is passed iff the outcomes of all $Z$ measurements coincide. Each of the other admissible tests is associated with a string $\mathbf{r} \in \mathbb{Z}_{d}^{n}$ with $\sum_{k} r_{k}=0 \bmod d$, which means party $k$ performs the measurement on the eigenbasis of $X Z^{r_{k}}$ for $k=1,2, \ldots, n$. The canonical test projector reads

$$
P_{\mathbf{r}}=\frac{1}{d} \sum_{l=0}^{d-1}\left(\prod_{k=1}^{n} X_{k} Z_{k}^{r_{k}}\right)^{l}
$$

Denote the outcome of party $k$ by an integer $o_{k} \in \mathbb{Z}_{d}$ corresponding to the eigenvalue $\omega^{o_{k}}$ of $X Z^{r_{k}}$; then the test is passed if $\sum_{k} o_{k}=0 \bmod d$, so that $\prod_{k=1}^{n} X_{k} Z_{k}^{r_{k}}$ has eigenvalue 1 . The following lemma is the analog of Lemma 1 for the qudit case; the proof is also similar and thus omitted.

Lemma 2. Suppose $d$ is an odd prime. Then the GHZ state $\left|\mathrm{G}_{n}^{d}\right\rangle$ has $1+d^{n-1}$ admissible Pauli measurements and $1+d^{n-1}$ admissible test operators. Except for the test projector $P_{0}$ in Eq. (16), all other admissible test operators have the form in Eq. (17) with $\sum_{k} r_{k}=0 \bmod d$.

Our verification protocol is composed of all $1+d^{n-1}$ admissible tests based on Pauli measurements. The test $P_{0}$ is performed with probability $1 /(d+1)$ and the other $d^{n-1}$ tests are performed with probability $1 /\left[(d+1) d^{n-2}\right]$ each. The resulting verification operator is homogeneous and has the form

$$
\Omega_{\mathrm{II}}:=\frac{1}{d+1}\left(P_{0}+\frac{1}{d^{n-2}} \sum_{\mathbf{r}} P_{\mathbf{r}}\right)=\frac{\mathbb{1}+d\left|\mathrm{G}_{n}^{d}\right\rangle\left\langle\mathrm{G}_{n}^{d}\right|}{d+1}
$$

where the second equality is proved in Appendix $B$. We have $\beta\left(\Omega_{\mathrm{II}}\right)=1 /(d+1)$, and

$$
\nu\left(\Omega_{\mathrm{II}}\right)=\frac{d}{d+1}, \quad N\left(\Omega_{\mathrm{II}}\right) \approx \frac{d+1}{d \varepsilon} \ln \delta^{-1} .
$$


Similar to the qubit case, this protocol is optimal among all protocols based on separable measurements. In addition, it is essentially the unique optimal protocol based on Pauli measurements; the number $1+d^{n-1}$ of measurement settings is the smallest possible. Proposition 2 below generalizes Proposition 1 to the qudit case. Its proof is a simple analog of the counterpart for the qubit case and is thus omitted. As in the qubit case, there is some freedom in choosing the Pauli group, and here we focus on the canonical Pauli group generated by the operators $Z$ and $X$ defined in Eq. (15) for each qudit.

Proposition 2. Suppose $\Omega$ is an optimal verification strategy with $\nu(\Omega)=d /(d+1)$ for $\left|\mathrm{G}_{n}^{d}\right\rangle$ that is based on Pauli measurements, where $d$ is an odd prime. Then $\Omega=\Omega_{\mathrm{II}}$; in addition, $\Omega$ is composed of admissible tests with the same probabilities as in $\Omega_{\mathrm{II}}$.

\section{Alternative optimal protocol based on 2-designs}

When the local dimension $d$ is not necessarily a prime, we can still devise optimal protocols for verifying GHZ states by virtue of (weighted complex projective) 2designs [39 41]. Let $\left\{\mathcal{B}_{h}\right\}_{h=0}^{m}$ be $m+1$ bases on the Hilbert space of dimension $d$, where $\mathcal{B}_{0}$ is the standard basis, and each basis $\mathcal{B}_{h}$ for $h=1,2, \ldots, m$ is composed of $d$ kets of the form

$$
\left|\psi_{h t}\right\rangle=\frac{1}{\sqrt{d}} \sum_{j=0}^{d-1} \mathrm{e}^{\mathrm{i} \theta_{h t j}}|j\rangle, \quad \theta_{h t j}=2 \pi\left[\frac{t j}{d}+\frac{h\left(\begin{array}{l}
j \\
2
\end{array}\right)}{m}\right]
$$

for $t \in \mathbb{Z}_{d}$. Let $w_{0}=1 /(d+1)$ and $w_{h}=d /[m(d+1)]$ for $h=1,2, \ldots, m$, and let $\left\{\mathcal{B}_{h}, w_{h}\right\}_{h=0}^{m}$ be a weighted set of kets with weight $w_{h}$ for all kets in basis $h$. When $d \geq 3$ and $m \geq\left\lceil\frac{3}{4}(d-1)^{2}\right\rceil$, the set $\left\{\mathcal{B}_{h}, w_{h}\right\}_{h=0}^{m}$ forms a 2-design according to Ref. 41]. Define

$$
W:=\operatorname{diag}\left(\mu^{0}, \mu^{1}, \ldots, \mu^{d-2}, \mu^{-(d-1)(d-2) / 2}\right),
$$

where $\mu=\mathrm{e}^{2 \pi \mathrm{i} / m}$ is a primitive $m$ th root of unity. Then $\left|\psi_{h t}\right\rangle$ is an eigenstate of $X W^{h}$ with eigenvalue $\omega^{-t}$ as shown in Appendix C that is,

$$
X W^{h}=\sum_{t \in \mathbb{Z}_{d}} \omega^{-t}\left|\psi_{h t}\right\rangle\left\langle\psi_{h t}\right| .
$$

When $d \geq 3$, by virtue of the 2-design $\left\{\mathcal{B}_{h}, w_{h}\right\}_{h=0}^{m}$ we can construct an optimal protocol using $1+m^{n-1}$ distinct tests. The first test is still the standard test $P_{0}$ as given in Eq. (16). Each of the other tests is specified by a string $\mathbf{h} \in\{1,2, \ldots, m\}^{n}$ with $\sum_{k} h_{k}=0 \bmod m$, which means party $k$ (for $k=1, \ldots, n$ ) performs the projective measurement on the basis $\mathcal{B}_{h_{k}}$. The outcome of party $k$ is denoted by $o_{k} \in \mathbb{Z}_{d}$, which corresponds to the ket $\left|\psi_{h_{k} o_{k}}\right\rangle$ and the eigenvalue $\omega^{-o_{k}}$ of $X W^{h}$. The test is passed if $\sum_{k} o_{k}=0 \bmod d$, and the test projector reads

$$
P_{\mathbf{h}}=\frac{1}{d} \sum_{l=0}^{d-1}\left(\prod_{k=1}^{n} X_{k} W_{k}^{h_{k}}\right)^{l}
$$

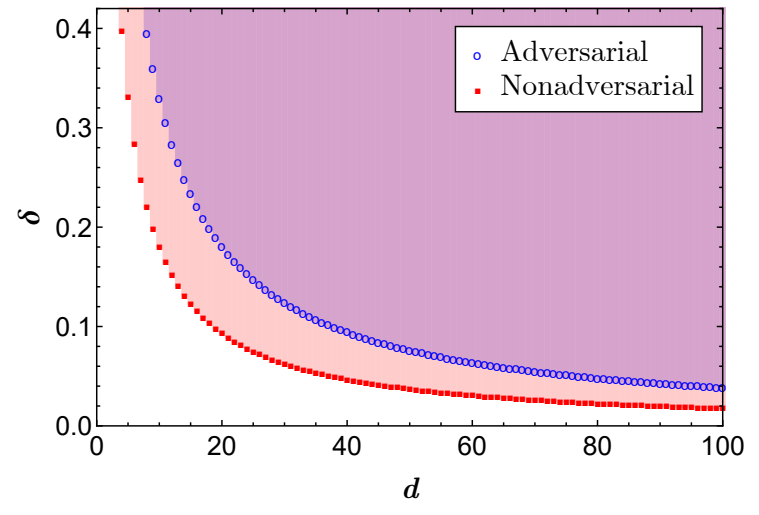

FIG. 1. Certification of the GME of the $n$-qudit GHZ state in the adversarial scenario and the nonadversarial scenario using only one test. Here $d$ is the local dimension; the significance level $\delta$ associated with the shaded region is achievable. The homogeneous strategy $\Omega$ with $\beta(\Omega)=1 /(d+1)$ $[\beta(\Omega)=2 /(d+1)]$ is applied to the nonadversarial scenario (adversarial scenario).

Note that all eigenvalues of $\prod_{k=1}^{n} X_{k} W_{k}^{h_{k}}$ are powers of $\omega$ according to Eq. 222, so $P_{\mathbf{h}}$ is the projector onto the eigenspace with eigenvalue 1 . In addition, the target state $\left|\mathrm{G}_{n}^{d}\right\rangle$ is stabilized by $\prod_{k=1}^{n} X_{k} W_{k}^{h_{k}}$ given the assumption $\sum_{k} h_{k}=0 \bmod m$ and so can pass the test with certainty as desired.

We perform the test $P_{0}$ with probability $1 /(d+1)$ and the other $m^{n-1}$ tests with probability $d /\left[(d+1) m^{n-1}\right]$ each. The verification operator reads [cf. $\Omega_{\mathrm{II}}$ in Eq. [18]]

$$
\Omega_{\mathrm{III}}:=\frac{1}{d+1}\left(P_{0}+\frac{d}{m^{n-1}} \sum_{\mathbf{h}} P_{\mathbf{h}}\right)=\frac{\mathbb{1}+d\left|\mathrm{G}_{n}^{d}\right\rangle\left\langle\mathrm{G}_{n}^{d}\right|}{d+1},
$$

where the second equality is proved in Appendix C This protocol is optimal among all protocols based on separable measurements. Compared with the protocol based on Pauli measurements, this protocol applies to GHZ states of any local dimension $d$ with $d \geq 3$, although it requires more measurement settings. In addition, this protocol is the only homogeneous protocol for general GHZ states beyond qubit systems.

\section{Efficient certification of GME}

A quantum state $\rho$ is genuinely multipartite entangled (i.e., GME) if its fidelity with the GHZ state $\operatorname{tr}\left(\rho\left|\mathrm{G}_{n}^{d}\right\rangle\left\langle\mathrm{G}_{n}^{d}\right|\right)$ is larger than $1 / d[3]$. To certify the GME of the qudit GHZ state with significance level $\delta$ using a given verification strategy $\Omega$, the number of tests is determined by Eq. (3) with $\varepsilon=(d-1) / d$. If, in addition, $\Omega$ is the optimal local strategy with $\nu(\Omega)=d /(d+1)$, then this number reads

$$
N_{\mathrm{E}}=\left\lceil\frac{\ln \delta}{\ln 2-\ln (d+1)}\right\rceil .
$$


We have $N_{\mathrm{E}}=1$ when $d \geq 2 \delta^{-1}-1$, so the GME of the GHZ state can be certified with any given significance level using only one test when the local dimension $d$ is sufficiently large, as illustrated in Fig. 1. Compared with previous approaches for detecting GME that are based on witness operators 42, 43, our approach requires much fewer measurements. Although single-copy entanglement detection is known before [32, 44, single-copy detection of GME is still quite surprising, because it is much more difficult to demonstrate GME than just entanglement.

In sharp contrast, the previous verification protocols proposed in Refs. 24, 29] cannot certify the GME of GHZ states using a single test whenever $\delta \leq 1 / 2$ (the parameter range of practical interest). To be specific, the strategy $\Omega_{\mathrm{PLM}}$ in Ref. 24] only applies to the qubit case and has a spectral gap $\nu=2^{n-1} /\left(2^{n}-1\right)$. To certify the GME with significance level $\delta$, the number of tests required reads

$$
N_{\mathrm{E}}\left(\Omega_{\mathrm{PLM}}\right)=\left\lceil\frac{\ln \delta}{\ln \left[1-2^{n-2} /\left(2^{n}-1\right)\right]}\right\rceil,
$$

so the GME cannot be certified using a single test when $\delta<5 / 7$ (for $n \geq 3$ ). The strategy $\Omega_{\mathrm{ZH}}$ in Ref. 29]. applies to the qudit case and has spectral gap $\nu=1 / 2$. The number of tests required reads

$$
N_{\mathrm{E}}\left(\Omega_{\mathrm{ZH}}\right)=\left\lceil\frac{\ln \delta}{\ln (d+1)-\ln (2 d)}\right\rceil,
$$

so the GME cannot be certified using a single test when $\delta \leq 1 / 2$, irrespective of the local dimension $d$. For example, to certify the GME of the GHZ state with significance level $\delta=0.01$ (0.001), the strategy $\Omega_{\mathrm{PLM}}$ in Ref. 24] requires at least 14 (21) tests, while the strategy $\Omega_{\mathrm{ZH}}$ in Ref. 29] requires at least 7 (10) tests. These observations demonstrate that our protocols are much more efficient than previous protocols for certifying GME.

\section{VERIFICATION OF GHZ-LIKE STATES}

Next, consider the GHZ-like states

$$
|\xi\rangle=\sum_{j=0}^{d-1} \lambda_{j}|j\rangle^{\otimes n}
$$

where the coefficients $\lambda_{j}$ have decreasing order $1>\lambda_{0} \geq$ $\lambda_{1} \geq \cdots \lambda_{d-1} \geq 0$ and satisfy $\sum_{j=0}^{d-1} \lambda_{j}^{2}=1$. Such states are of interest to quantum state sharing [45] and foundational studies on nonlocality [46, 47. They are also useful in improving signal-to-noise ratios in interferometry [48] and enhancing signal amplitudes of the electronic spin readout [49].

\section{A. Simplest protocol for verifying GHZ-like states}

We first show that the GHZ-like state $|\xi\rangle$ can be verified efficiently using only two distinct tests con- structed from mutually unbiased bases (MUB). Recall that two bases $\left\{\left|\psi_{i}\right\rangle\right\}_{i=0}^{d-1}$ and $\left\{\left|\varphi_{j}\right\rangle\right\}_{j=0}^{d-1}$ for a Hilbert space of dimension $d$ are mutually unbiased if they satisfy $\left|\left\langle\psi_{i} \mid \varphi_{j}\right\rangle\right|^{2}=1 / d$ for all $i$ and $j\left[50-52\right.$. Let $\mathcal{B}_{0}$ be the standard computational basis and let $\mathcal{B}=\left\{\left|u_{g}\right\rangle\right\}_{g \in \mathbb{Z}_{d}}$ be any basis that is unbiased with $\mathcal{B}_{0}$. A simple example of $\mathcal{B}$ is the Fourier basis $\left\{\sum_{j=0}^{d-1} \omega^{g j}|j\rangle / \sqrt{d}\right\}_{g \in \mathbb{Z}_{d}}$ with $\omega=\mathrm{e}^{2 \pi \mathrm{i} / d}$, which happens to be the eigenbasis of the shift operator $X$ in Eq. (15). The following discussion is independent of the choice of the basis $\mathcal{B}$ as long as it is unbiased with respect to the standard basis $\mathcal{B}_{0}$.

The first test is the standard test $P_{0}$ in Eq. (16). For the second test, the first $n-1$ parties perform projective measurements on the basis $\mathcal{B}$. If they obtain the outcome $\mathbf{g}=\left\{g_{1}, g_{2}, \ldots, g_{n-1}\right\} \in \mathbb{Z}_{d}^{n-1}$, then the normalized reduced state of party $n$ reads

$$
d^{\frac{n-1}{2}}\left(\bigotimes_{k=1}^{n-1}\left\langle u_{g_{k}}\right|\right)|\xi\rangle=M\left|v_{\mathbf{g}}\right\rangle
$$

where

$$
\begin{aligned}
\left|v_{\mathbf{g}}\right\rangle & :=d^{\frac{n-1}{2}}\left(\bigotimes_{k=1}^{n-1}\left\langle u_{g_{k}}\right|\right)\left|\mathrm{G}_{n}^{d}\right\rangle, \\
M & :=\sqrt{d} \operatorname{diag}\left(\lambda_{0}, \lambda_{1}, \ldots, \lambda_{d-1}\right) .
\end{aligned}
$$

Note that $\left|v_{\mathbf{g}}\right\rangle$ has a constant overlap of $1 / d$ with each element in the basis $\mathcal{B}_{0}$. Then party $n$ performs the projective measurement $\left\{M\left|v_{\mathbf{g}}\right\rangle\left\langle v_{\mathbf{g}}|M, I-M| v_{\mathbf{g}}\right\rangle\left\langle v_{\mathbf{g}}\right| M\right\}$, where $I$ is the identity operator on the Hilbert space of one qudit. The test is passed if party $n$ obtains the first outcome (corresponding to $M\left|v_{\mathbf{g}}\right\rangle\left\langle v_{\mathbf{g}}\right| M$ ). The resulting test projector reads

$$
P_{1}=\sum_{\mathbf{g}}\left[\bigotimes_{k=1}^{n-1}\left(\left|u_{g_{k}}\right\rangle\left\langle u_{g_{k}}\right|\right)\right] \otimes\left(M\left|v_{\mathbf{g}}\right\rangle\left\langle v_{\mathbf{g}}\right| M\right)
$$

So we have

$$
\operatorname{tr}\left(P_{0} P_{1}\right)=\frac{1}{d^{n-1}} \sum_{\mathbf{g}} \sum_{j=0}^{d-1}\left|\left\langle j|M| v_{\mathbf{g}}\right\rangle\right|^{2}=\frac{1}{d^{n-1}} \sum_{\mathbf{g}, j} \lambda_{j}^{2}=1,
$$

which implies that the two projectors $\bar{P}_{0}:=P_{0}-|\xi\rangle\langle\xi|$ and $\bar{P}_{1}:=P_{1}-|\xi\rangle\langle\xi|$ have orthogonal supports.

If we perform the two tests $P_{0}$ and $P_{1}$ with probability $p$ and $1-p$, respectively, then the verification operator reads $\Omega_{\mathrm{IV}}=p P_{0}+(1-p) P_{1}$, with

$$
\beta\left(\Omega_{\mathrm{IV}}\right)=\left\|\bar{\Omega}_{\mathrm{IV}}\right\|=\max \{p, 1-p\} \geq \frac{1}{2},
$$

where $\bar{\Omega}_{\mathrm{IV}}=\Omega_{\mathrm{IV}}-|\xi\rangle\langle\xi|$. The lower bound is saturated iff $p=1 / 2$, in which case we have $\Omega_{\mathrm{IV}}=\left(P_{0}+P_{1}\right) / 2$. The corresponding spectral gap $\nu\left(\Omega_{\mathrm{IV}}\right)$ and the number $N\left(\Omega_{\mathrm{IV}}\right)$ of required tests read

$$
\nu\left(\Omega_{\mathrm{IV}}\right)=\frac{1}{2}, \quad N\left(\Omega_{\mathrm{IV}}\right) \approx \frac{2}{\varepsilon} \ln \delta^{-1} .
$$


According to Ref. 32, here the spectral gap attains the maximum among all protocols composed of two distinct local projective tests, so the above protocol is the most efficient among all protocols based on two distinct local projective tests.

\section{B. Optimal protocol under one-way LOCC}

For a bipartite state $|\zeta\rangle=\sum_{j=0}^{d-1} \lambda_{j}|j j\rangle$ with the same local dimension and coefficients $\lambda_{j}$ as $|\xi\rangle$ in Eq. (28), the maximum spectral gap of any verification operator based on one-way LOCC is $1 /\left(1+\lambda_{0}^{2}\right)$ [34, 35]. The counterpart for the GHZ-like state $|\xi\rangle$ cannot be larger. Here we shall demonstrate that this upper bound can be saturated. When $d \geq 3$, our protocol consists of $1+m^{n-1}$ distinct tests with $m \geq\left\lceil\frac{3}{4}(d-1)^{2}\right\rceil$. The first one is the standard test in Eq. (16). For each of the other tests, the first $n-1$ parties perform projective measurements on the bases $\mathcal{B}_{h_{1}}, \mathcal{B}_{h_{2}}, \ldots, \mathcal{B}_{h_{n-1}}[\mathrm{cf}$. Eq. 20]], respectively, where $h_{1}, h_{2}, \ldots, h_{n-1} \in\{1,2, \ldots, m\}$. After receiving the outcomes $o_{1}, o_{2}, \ldots, o_{n-1} \in \mathbb{Z}_{d}$ of these measurements, we choose $h_{n}, o_{n}$ to satisfy the conditions $\sum_{k=1}^{n} h_{k}=0$ $\bmod m$ and $\sum_{k=1}^{n} o_{k}=0 \bmod d$. Then party $n$ performs the projective measurement $\left\{M P_{h_{n} o_{n}} M, I-M P_{h_{n} o_{n}} M\right\}$, where $P_{h_{k} o_{k}}=\left|\psi_{h_{k} o_{k}}\right\rangle\left\langle\psi_{h_{k} o_{k}}\right|$ and $M$ is defined in Eq. (31). The test is passed if party $n$ obtains the first outcome (corresponding to $M P_{h_{n} o_{n}} M$ ), and the test projector reads

$$
P_{\mathbf{h}}^{\prime}=\left(I^{\otimes(n-1)} \otimes M\right) P_{\mathbf{h}}\left(I^{\otimes(n-1)} \otimes M\right),
$$

where $P_{\mathbf{h}}$ is the test projector in Eq. (23).

Suppose we perform the test $P_{0}$ with probability $p$ and each of the other tests with probability $(1-p) / m^{n-1}$; then the verification operator reads

$$
\Omega_{\mathrm{V}}=p P_{0}+(1-p) \Pi
$$

where

$$
\Pi:=\frac{\sum_{\mathbf{h}} P_{\mathbf{h}}^{\prime}}{m^{n-1}}=|\xi\rangle\langle\xi|+I^{\otimes(n-1)} \otimes \rho_{n}-\sum_{j=0}^{d-1} \lambda_{j}^{2}(|j\rangle\langle j|)^{\otimes n},
$$

with $\rho_{n}=\operatorname{tr}_{1,2, \ldots, n-1}(|\xi\rangle\langle\xi|)=\sum_{j=0}^{d-1} \lambda_{j}^{2}|j\rangle\langle j|$ being the reduced state for party $n$. Here the second equality follows from Eqs. 24) and (36) [cf. Eq. C4) in Appendix C]. Note that $\bar{\Pi}=\bar{\Pi}-|\xi\rangle\left\langle\bar{\xi}\right.$ and $\left.\bar{P}_{0}=P_{0}-\mid \xi\right\rangle\langle\xi|$ are orthogonal; we conclude that

$$
\begin{aligned}
\beta\left(\Omega_{\mathrm{V}}\right) & =\left\|\bar{\Omega}_{\mathrm{V}}\right\|=\max \{p,(1-p)\|\bar{\Pi}\|\} \\
& =\max \left\{p,(1-p) \lambda_{0}^{2}\right\} \geq \frac{\lambda_{0}^{2}}{1+\lambda_{0}^{2}} .
\end{aligned}
$$

The bound is saturated iff $p=\lambda_{0}^{2} /\left(1+\lambda_{0}^{2}\right)$, in which case we have

$$
\nu\left(\Omega_{\mathrm{V}}\right)=\frac{1}{1+\lambda_{0}^{2}}, \quad N\left(\Omega_{\mathrm{V}}\right) \approx \frac{1+\lambda_{0}^{2}}{\varepsilon} \ln \delta^{-1} .
$$

Therefore, this protocol is optimal among all protocols based on one-way LOCC.

When the local dimension $d$ is a prime, the number of distinct tests required for constructing the optimal protocol can be reduced to $1+d^{n-1}$. Take the qubit case for example. The first test is still the standard test $P_{0}$. For each of the other tests, the first $n-1$ parties perform either $X$ or $Y$ measurements. Then party $n$ performs the projective measurement $\{|v\rangle\langle v|, I-| v\rangle\langle v|\}$, where $|v\rangle$ is the normalized reduced state of party $n$ depending on the outcomes of the first $n-1$ parties. The test is passed if party $n$ obtains the first outcome (corresponding to $|v\rangle\langle v|)$. The test projector has the form

$$
P_{\mathscr{Y}}^{\prime}=\left(I^{\otimes(n-1)} \otimes M\right) P_{\mathscr{Y}}\left(I^{\otimes(n-1)} \otimes M\right),
$$

where $\mathscr{Y} \subset\{1,2, \ldots, n\}$ has even cardinality and $P_{\mathscr{Y}}$ is the test projector in Eq. (12). Suppose we perform the test $P_{0}$ with probability $p$ and each of the other tests with probability $(1-p) / 2^{n-1}$; then the verification operator reads

$$
\Omega_{\mathrm{V}}^{\prime}=p P_{0}+\frac{1-p}{2^{n-1}} \sum_{\mathscr{Y}} P_{\mathscr{Y}}^{\prime} .
$$

Again, the maximum spectral gap $\nu\left(\Omega_{\mathrm{V}}^{\prime}\right)=1 /\left(1+\lambda_{0}^{2}\right)$ is attained when $p=\lambda_{0}^{2} /\left(1+\lambda_{0}^{2}\right)$. When $d$ is an odd prime, more details can be found in Appendix $D$.

\section{Improved protocol based on more communications}

The above protocol for verifying GHZ-like states can be improved further if more communications are allowed. Let $\Omega_{k}(k=1,2, \ldots, n)$ be the strategy defined according to Eq. (37), but with the roles of party $k$ and party $n$ interchanged; that is, the measurement performed by party $k$ depends on the measurement outcomes of the other $n-1$ parties. Then we can construct a new strategy by applying $\Omega_{1}, \Omega_{2}, \ldots, \Omega_{n}$ with probability $1 / n$ each, and the resulting verification operator reads

$$
\Omega_{\mathrm{VI}}=\frac{1}{n} \sum_{k=1}^{n} \Omega_{k}=p P_{0}+(1-p) \frac{1}{n} \sum_{k=1}^{n} \Pi_{k}
$$

Here the operator $\Pi_{k}$ is derived from $\Pi$ in Eq. (38) by replacing $I^{\otimes(n-1)} \otimes \rho_{n}$ with $R_{k}:=I^{\otimes(k-1)} \otimes \rho_{k} \otimes I^{\otimes(n-k)}$, where $\rho_{k}=\sum_{j=0}^{d-1} \lambda_{j}^{2}|j\rangle\langle j|$. We have

$$
\begin{gathered}
\frac{1}{n} \sum_{k=1}^{n} \Pi_{k}=|\xi\rangle\langle\xi|+\frac{1}{n} \sum_{k=1}^{n} R_{k}-\sum_{j=0}^{d-1} \lambda_{j}^{2}(|j\rangle\langle j|)^{\otimes n}, \\
\| \frac{1}{n} \sum_{k=1}^{n} \Pi_{k}-|\xi\rangle\langle\xi| \|=\frac{(n-1) \lambda_{0}^{2}+\lambda_{1}^{2}}{n} .
\end{gathered}
$$

Therefore,

$$
\begin{aligned}
\beta\left(\Omega_{\mathrm{VI}}\right) & =\max \left\{p,(1-p) n^{-1}\left[(n-1) \lambda_{0}^{2}+\lambda_{1}^{2}\right]\right\} \\
& \geq\left[n+(n-1) \lambda_{0}^{2}+\lambda_{1}^{2}\right]^{-1}\left[(n-1) \lambda_{0}^{2}+\lambda_{1}^{2}\right] .
\end{aligned}
$$


The bound is saturated when

$$
p=\frac{(n-1) \lambda_{0}^{2}+\lambda_{1}^{2}}{n+(n-1) \lambda_{0}^{2}+\lambda_{1}^{2}}
$$

in which case we have

$$
\nu\left(\Omega_{\mathrm{VI}}\right)=\frac{n}{n+(n-1) \lambda_{0}^{2}+\lambda_{1}^{2}} \geq \nu\left(\Omega_{\mathrm{V}}\right) .
$$

The strategy $\Omega_{\mathrm{VI}}$ is more efficient than $\Omega_{\mathrm{V}}$ except when $\lambda_{1}=\lambda_{0}$, as illustrated in Fig. 2 .

\section{ADVERSARIAL SCENARIO}

Finally, we turn to the adversarial scenario, in which the quantum device is controlled by a potentially malicious adversary, and can produce an arbitrary correlated or entangled state $\rho$ on the whole system $\mathcal{H}^{\otimes(N+1)}$ [27. [53. By virtue of a general recipe proposed in Refs. 25, 26, we can verify the target state $|\Psi\rangle$ in the adversarial scenario by first randomly choosing $N$ systems and then applying a verification strategy $\Omega$ to each system chosen. Note that only one-way communication from the adversary to the verifier is involved. In addition, usually the choices of the $N$ systems and the specific test for each system chosen can be determined after receiving the state $\rho$. Therefore, the adversary has no information about these choices before sending the state $\rho$, and he/she cannot get any advantage even if these choices are broadcast after sending the state $\rho$. By constructing a suitable strategy $\Omega$, we can make sure with high confidence (low significance level) that the reduced state on the remaining system has fidelity at least $1-\varepsilon$ if all $N$ tests are passed. Efficient state verification in such an adversarial scenario is crucial to quantum secret sharing [4, 5] and quantum networks [8.

If there is no restriction on the accessible measurements, then the optimal strategy can be chosen to be homogeneous 25, 26. In the high-precision limit $\varepsilon, \delta \rightarrow 0$, the minimal number of tests required to verify $|\Psi\rangle$ within infidelity $\varepsilon$ and significance level $\delta$ reads [25, 26] (assum$\operatorname{ing} \beta(\Omega)>0)$

$$
N \approx\left[\beta(\Omega) \varepsilon \ln \beta(\Omega)^{-1}\right]^{-1} \ln \delta^{-1} .
$$

This number is minimized when $\beta(\Omega)=1 / \mathrm{e}$, which yields $N \approx \mathrm{e} \varepsilon^{-1} \ln \delta^{-1}$. In addition, this number increases monotonically when $\beta(\Omega)$ deviates from the value $1 /$ e. If $\varepsilon, \delta$ are small but not infinitesimal, say $\varepsilon, \delta \leq 0.01$, then the choice $\beta(\Omega)=1 / \mathrm{e}$ is nearly optimal even if it is not exactly optimal.

Our strategies for verifying the qudit GHZ state $\left|\mathrm{G}_{n}^{d}\right\rangle$ are homogeneous with $\beta(\Omega)=1 /(d+1)$. To construct the optimal verification strategy in the adversarial scenario, it suffices to add the trivial test with a suitable probability $p$. The test operator associated with the trivial test is the identity operator, so all states can pass the test for

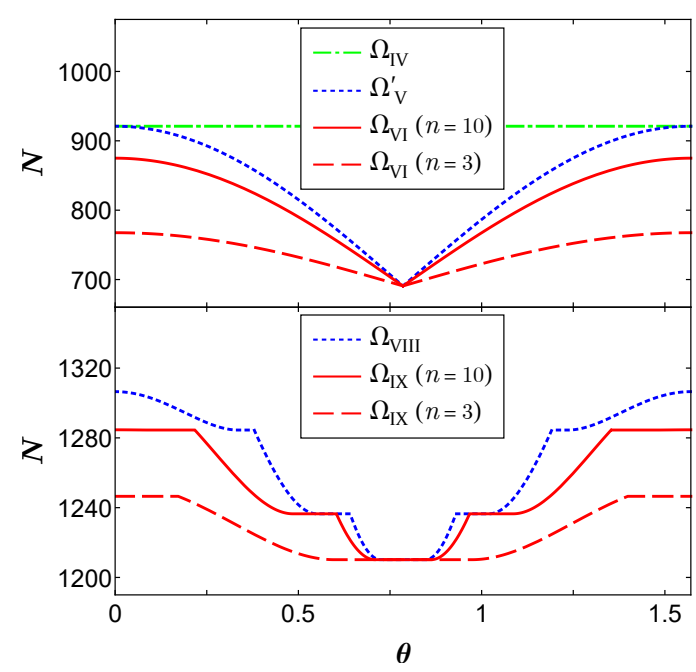

FIG. 2. Efficient verification of the $n$-qubit GHZ-like state $|\xi\rangle=\cos \theta|0\rangle^{\otimes n}+\sin \theta|1\rangle^{\otimes n}$ in the nonadversarial scenario (upper plot) and the adversarial scenario (lower plot). Here $N$ is the number of tests required to achieve infidelity $\varepsilon=0.01$ and significance level $\delta=0.01$. Note that $N\left(\Omega_{\mathrm{VI}}\right)$ and $N\left(\Omega_{\mathrm{IX}}\right)$ are dependent on the qubit number $n$, while $N\left(\Omega_{\mathrm{IV}}\right), N\left(\Omega_{\mathrm{V}}^{\prime}\right)$, and $N\left(\Omega_{\text {VIII }}\right)$ are not.

sure. Let $p=[(d+1) \beta-1] / d$ with $1 /(d+1) \leq \beta<1$; then the verification operator reads

$$
\begin{aligned}
\Omega_{\mathrm{VII}} & :=(1-p) \frac{\mathbb{1}+d\left|\mathrm{G}_{n}^{d}\right\rangle\left\langle\mathrm{G}_{n}^{d}\right|}{d+1}+p \mathbb{1} \\
& =\left|\mathrm{G}_{n}^{d}\right\rangle\left\langle\mathrm{G}_{n}^{d}\right|+\beta\left(\mathbb{1}-\left|\mathrm{G}_{n}^{d}\right\rangle\left\langle\mathrm{G}_{n}^{d}\right|\right) .
\end{aligned}
$$

Any homogeneous strategy $\Omega$ with $1 /(d+1) \leq \beta(\Omega)<1$ can be so constructed using local projective measurements. In particular, by choosing $p=(d+1-\mathrm{e}) /(\mathrm{e} d)$, we can construct the homogeneous strategy $\Omega_{\mathrm{VII}}$ with $\beta\left(\Omega_{\mathrm{VII}}\right)=1 / \mathrm{e}$, which is optimal for high-precision verification in the adversarial scenario (the optimal value may be slightly different when $\varepsilon, \delta$ are small but not infinitesimal). Similarly, we can construct a homogeneous strategy $\Omega$ with $\beta(\Omega)=2 /(d+1)$, with which the GME can be certified in the adversarial scenario using only one test as long as the significance level satisfies $\delta \geq 4 d /(d+1)^{2}$, as illustrated in Fig. 1. This claim follows from Corollary 6 in Ref. [26] with $\varepsilon=(d-1) / d$ (see also Theorem 3 in Ref. [32]). In sharp contrast, previous protocols in Refs. [24, 29] cannot certify the GME using a single test whenever $\delta \leq 1 / 2$ even in the nonadversarial scenario (cf. Sec. [IID), not to mention the adversarial scenario.

Next, we devise a homogeneous strategy for verifying the GHZ-like state $|\xi\rangle$ in Eq. (28) by modifying the strategy $\Omega_{\mathrm{V}}$ in Eq. (37), which requires one-way communication. Let $\lambda_{0}^{2} /\left(1+\lambda_{0}^{2}\right) \leq p<1$ and replace the test projector $P_{0}$ in Eq. (16) with the following test operator

$$
Q_{0}=P_{0}+\sum_{\mathbf{j} \in \mathscr{B}}\left[1-\left(\frac{1}{p}-1\right) \lambda_{j_{n}}^{2}\right]|\mathbf{j}\rangle\langle\mathbf{j}|
$$


where $\mathscr{B}$ denotes the subset of $\mathbb{Z}_{d}^{n}$ excluding elements $\mathbf{j}$ that satisfy $j_{1}=j_{2}=\cdots=j_{n}$. Note that $Q_{0}$ can be realized by local projective measurements: All $n$ parties perform projective measurements on the standard basis; the test is passed with certainty if they obtain the same outcome, while with probability $1-\left(p^{-1}-1\right) \lambda_{j_{n}}^{2}$ if they do not obtain the same outcome and party $n$ obtains outcome $j_{n}$. Then the verification operator $\Omega_{\mathrm{V}}$ turns into

$$
\Omega_{\mathrm{VIII}}=p Q_{0}+(1-p) \Pi=|\xi\rangle\langle\xi|+p(\mathbb{1}-|\xi\rangle\langle\xi|),
$$

which is homogeneous with $\beta(\Omega)=p$. Here $\Pi$ is defined in Eq. (38). To achieve optimal performance in highprecision verification in the adversarial scenario, we can choose $p=\max \left\{\mathrm{e}^{-1}, \lambda_{0}^{2} /\left(1+\lambda_{0}^{2}\right)\right\}$. If $\lambda_{0}^{2} \leq 1 /(\mathrm{e}-1)$, then we have $\beta(\Omega)=1 / \mathrm{e}$, so the homogeneous strategy $\Omega_{\text {VIII }}$ constructed in this way is optimal even among strategies that can access entangling measurements. In general, $\Omega_{\mathrm{VIII}}$ is optimal among all strategies based on one-way LOCC. Even in the worst case with $\beta(\Omega)=1 / 2$, the number of required tests is only $2\left(\ln \delta^{-1}\right) /(\varepsilon \ln 2)$, and the overhead compared with the optimal strategy based on entangling measurements is only about $6 \%$. By contrast, the choice $p=\lambda_{0}^{2} /\left(1+\lambda_{0}^{2}\right)$ is optimal for fidelity estimation.

The strategy $\Omega_{\mathrm{VI}}$ in Eq. 43 can also be turned into a homogeneous strategy. Let

$$
\frac{(n-1) \lambda_{0}^{2}+\lambda_{1}^{2}}{n+(n-1) \lambda_{0}^{2}+\lambda_{1}^{2}} \leq p<1
$$

and replace the projector $P_{0}$ with the following operator

$$
\tilde{Q}_{0}=P_{0}+\sum_{\mathbf{j} \in \mathscr{B}}\left[1-\frac{1}{n}\left(\frac{1}{p}-1\right) \sum_{k=1}^{n} \lambda_{j_{k}}^{2}\right]|\mathbf{j}\rangle\langle\mathbf{j}|,
$$

which can be realized by local projective measurements in analogy to $Q_{0}$. The resulting verification operator reads

$$
\Omega_{\mathrm{IX}}=p \tilde{Q}_{0}+(1-p) \frac{1}{n} \sum_{k=1}^{n} \Pi_{k}=|\xi\rangle\langle\xi|+p(\mathbb{1}-|\xi\rangle\langle\xi|),
$$

which is homogeneous with $\beta(\Omega)=p$. For high-precision verification in the adversarial scenario, the optimal choice of $p$ is

$$
p=\max \left\{\mathrm{e}^{-1}, \frac{(n-1) \lambda_{0}^{2}+\lambda_{1}^{2}}{n+(n-1) \lambda_{0}^{2}+\lambda_{1}^{2}}\right\} .
$$

The resulting strategy $\Omega_{\mathrm{IX}}$ is optimal if

$$
(n-1) \lambda_{0}^{2}+\lambda_{1}^{2} \leq \frac{n}{\mathrm{e}-1},
$$

in which case we have $\beta(\Omega)=1 /$ e. For fidelity estimation, the optimal choice of $p$ coincides with the lower bound in Eq. (53), that is,

$$
\frac{(n-1) \lambda_{0}^{2}+\lambda_{1}^{2}}{n+(n-1) \lambda_{0}^{2}+\lambda_{1}^{2}}
$$

Although a lot of random bits are required to construct the above verification protocols, they can be generated by classical random number generators. In the adversarial scenario we consider, the adversary controls the preparation of quantum states, but not the measurement devices used to verify these states, which is in sharp contrast with self-testing [26, 54, 55]. In addition, there is only one-way communication from the adversary to the verifier. Usually the choices of the systems for verification/application and the specific test for each system chosen can be determined after the state is received from the adversary as mentioned in the beginning of this section. Therefore, even pseudo-random number generators like computer programs are sufficient for this task. Quantum resources are not necessary to generate random bits.

\section{SUMMARY}

We proposed optimal and homogeneous strategies for verifying GHZ states based on local projective measurements. Only Pauli measurements are required when the local dimension is a prime. These protocols are also surprisingly efficient for estimating the fidelity and certifying the GME. In particular, they enable the certification of the GME with any given significance level using only one test when the local dimension is sufficiently large. Such a high efficiency has never been achieved or even anticipated before. Our results indicate that it is easier to certify GME than thought previously. We hope that these results will be demonstrated in experiments in the near future. Moreover, our protocols can be generalized to verify GHZ-like states and can be applied to the adversarial scenario, while retaining a high efficiency. Our study provides an efficient tool for evaluating the qualities of GHZ states and GHZ-like states prepared in the lab. Meanwhile, it offers valuable insights into the verification, fidelity estimation, and entanglement certification of multipartite quantum states. In addition, the concepts of admissible measurements/test operators and canonical test projectors we introduced are useful beyond the focus of this work. In the future it would be desirable to generalize our results to other important multipartite quantum states.

\section{ACKNOWLEDGMENT}

HZ is grateful to Masahito Hayashi for discussions. This work is supported by the National Natural Science Foundation of China (Grant No. 11875110).

\section{Appendix A: Proofs of Lemma 1 and Proposition 1}

Proof of Lemma 1. To determine admissible Pauli measurements, we need to consider canonical test projectors associated with Pauli measurements. First note that an 
incomplete Pauli measurement cannot be admissible. To see this, it suffices to consider the case in which the incomplete Pauli measurement has weight $n-1$. After $n-1$ single-qubit Pauli measurements, the reduced states of the remaining party for all possible outcomes are eigenstates of one Pauli operator, so we can obtain a smaller canonical test projector by performing a suitable Pauli measurement on the remaining qubit. Therefore, it suffices to consider canonical test projectors associated with complete Pauli measurements.

Denote by $\mathscr{X}, \mathscr{Y}, \mathscr{Z}$ the sets of parties that perform $X, Y, Z$ measurements, respectively. If $|\mathscr{Z}| \geq 1$, then the canonical test projector reads

$$
\mathbb{1} \mathscr{X} \cup \mathscr{Y} \otimes\left[\bigotimes_{j \in \mathscr{Z}}(|0\rangle\langle 0|)_{j}+\bigotimes_{j \in \mathscr{Z}}(|1\rangle\langle 1|)_{j}\right] \geq P_{0}
$$

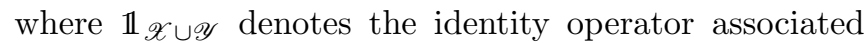
with parties in $\mathscr{X} \cup \mathscr{Y}$, and the subscript $j$ specifies the party on which the operators $|0\rangle\langle 0|$ and $|1\rangle\langle 1|$ act. The inequality in Eq. A1) is saturated iff all parties perform $Z$ measurements, that is, $|\mathscr{Z}|=n$. Note that every test projector of $\left|\mathrm{G}_{n}^{2}\right\rangle$ that is based on a Pauli measurement has rank at least 2 , so the test projector $P_{0}$ and the corresponding Pauli measurement are admissible, while other Pauli measurements with $|\mathscr{Z}| \geq 1$ and corresponding test projectors are not admissible.

Next, suppose each party performs either $X$ or $Y$ measurement, that is, $|\mathscr{Z}|=0$ and $|\mathscr{X}|+|\mathscr{Y}|=n$. If $|\mathscr{Y}|$ is odd, then the canonical test projector is equal to the identity and so cannot be admissible, given that all states in the measurement basis have nonzero overlaps with $\left|\mathrm{G}_{n}^{2}\right\rangle$. If $|\mathscr{Y}|$ is even, then the canonical test projector is $P_{\mathscr{Y}}$ given in Eq. (12), which has rank $2^{n-1}$. Note that $P_{\mathscr{Y}} \ngtr P_{0}$, and there is no other canonical test projector that is smaller than $P_{\mathscr{Y}}$. Therefore, all test projectors $P_{\mathscr{Y}}$ with even $|\mathscr{Y}|$ and corresponding Pauli measurements are admissible.

In summary, there are $1+2^{n-1}$ admissible Pauli measurements, namely, $Z^{n}$ and all strings in $\{X, Y\}^{n}$ with even numbers of $Y$. The corresponding $1+2^{n-1}$ canonical test projectors in Eqs. (11) and (12) are the only admissible test operators.

Proof of Proposition 1. To start with, suppose the verification strategy $\Omega$ only consists of admissible test projectors. According to Lemma $1, \Omega$ can be expressed as

$$
\Omega=p_{0} P_{0}+\sum_{\mathscr{Y}} p_{\mathscr{Y}} P_{\mathscr{Y}}, \quad p_{0}, p_{\mathscr{Y}} \geq 0, p_{0}+\sum_{\mathscr{Y}} p_{\mathscr{Y}}=1 .
$$

Now the assumption $\nu(\Omega)=2 / 3$ sets an upper bound for $p_{0}$, that is, $p_{0} \leq \beta(\Omega)=1 / 3$. Therefore,

$$
\operatorname{tr}(\Omega)=2 p_{0}+2^{n-1} \sum_{\mathscr{Y}} p_{\mathscr{Y}}=2 p_{0}+2^{n-1}\left(1-p_{0}\right) \geq \frac{2^{n}+2}{3}
$$

where the inequality is saturated iff $p_{0}=1 / 3$. In addition,

$$
\beta(\Omega) \geq \frac{\operatorname{tr}(\Omega)-1}{2^{n}-1} \geq \frac{2^{n}-1}{3\left(2^{n}-1\right)}=\frac{1}{3} .
$$

The first inequality is saturated iff $\Omega$ is homogeneous, which means all eigenvalues of $\Omega$ are equal except for the largest one. The second inequality is saturated iff the inequality in Eq. A3 is saturated, which implies that $p_{0}=1 / 3$. If $\nu(\Omega)=2 / 3$, that is, $\beta(\Omega)=1 / 3$, then both inequalities are saturated, so that

$$
\begin{aligned}
\Omega & =\frac{1}{3}\left(\mathbb{1}+2\left|\mathrm{G}_{n}^{2}\right\rangle\left\langle\mathrm{G}_{n}^{2}\right|\right) \\
& =\Omega_{\mathrm{I}}=\frac{1}{3}\left(P_{0}+\frac{1}{2^{n-2}} \sum_{\mathscr{Y}} P_{\mathscr{Y}}\right) .
\end{aligned}
$$

Moreover, the decomposition in the last expression is unique because the $1+2^{n-1}$ admissible canonical test projectors are linearly independent in the operator space.

If $\Omega$ consists of some nonadmissible test operators, we can construct a new strategy $\Omega^{\prime}$ by replacing each nonadmissible test operator $E$ with an admissible test projector $P$ satisfying $P \leq E$ and $\operatorname{tr}(P)<\operatorname{tr}(E)$. Then $\Omega^{\prime} \leq \Omega$ is an optimal strategy with $\nu\left(\Omega^{\prime}\right)=2 / 3$, which implies that $\Omega^{\prime}=\Omega_{\mathrm{I}}$ according to the above discussion. In addition, we have $\nu(\Omega)<\nu\left(\Omega^{\prime}\right)=2 / 3$ since $\Omega^{\prime}$ is homogeneous and $\operatorname{tr}\left(\Omega^{\prime}\right)<\operatorname{tr}(\Omega)$. This conclusion contradicts the assumption that $\nu(\Omega)=2 / 3$, which completes the proof of Proposition 1 .

Incidentally, Proposition 2 can be proved using a similar reasoning used in the proof of Proposition [1. Accordingly, Lemma 1 featuring in the above proof can be replaced by Lemma 2, which applies to the qudit case, assuming that $d$ is an odd prime.

\section{Appendix B: Proofs of Eqs. (13) and (18)}

Proof of Eq. (13). Note that the sum of all test projectors $P_{\mathscr{Y}}$ with $\mathscr{Y} \subset\{1, \ldots, n\}$ of even cardinalities can be expressed as

$$
\begin{aligned}
\sum_{\mathscr{Y}} P_{\mathscr{Y}} & =2^{n-2} \mathbb{1}+\frac{1}{2} \sum_{t=0}^{\lfloor n / 2\rfloor}(-1)^{t} \sum_{j} \mathcal{P}_{j}\left\{Y^{\otimes 2 t} \otimes X^{\otimes(n-2 t)}\right\} \\
& =2^{n-2} \mathbb{1}+\frac{1}{4}\left[(X+\mathrm{i} Y)^{\otimes n}+(X-\mathrm{i} Y)^{\otimes n}\right] \\
& =2^{n-2}\left[\mathbb{1}+(|0\rangle\langle 1|)^{\otimes n}+(|1\rangle\langle 0|)^{\otimes n}\right],
\end{aligned}
$$

where $\sum_{j} \mathcal{P}_{j}\left\{Y^{\otimes 2 t} \otimes X^{\otimes(n-2 t)}\right\}$ denotes the sum over $\left(\begin{array}{c}n \\ 2 t\end{array}\right)$ distinct permutations of $Y^{\otimes 2 t} \otimes X^{\otimes(n-2 t)}$. This equation implies the second equality in Eq. 13.

Proof of Eq. 18. The sum of all test projectors $P_{\mathbf{r}}$ with 
$\sum_{k} r_{k}=0 \bmod d$ can be expressed as

$$
\begin{aligned}
\sum_{\mathbf{r}} P_{\mathbf{r}} & =d^{n-2} \mathbb{1}+\frac{1}{d} \sum_{l=1}^{d-1} \sum_{\mathbf{r}} \prod_{k=1}^{n}\left(X_{k} Z_{k}^{r_{k}}\right)^{l} \\
& =d^{n-2} \mathbb{1}+\frac{1}{d^{2}} \sum_{l=1}^{d-1} \sum_{s \in \mathbb{Z}_{d}}\left[\sum_{r \in \mathbb{Z}_{d}} \omega^{-s r}\left(X Z^{r}\right)^{l}\right]^{\otimes n} \\
& =d^{n-2} \mathbb{1}+\frac{1}{d^{2}} \sum_{l=1}^{d-1} \sum_{j=0}^{d-1}(d|j+l\rangle\langle j|)^{\otimes n} \\
& =d^{n-2}\left[\mathbb{1}+\sum_{j^{\prime} \neq j}\left(\left|j^{\prime}\right\rangle\langle j|\right)^{\otimes n}\right]
\end{aligned}
$$

which implies Eq. 18). The first equality is meaningful when $d$ is odd, in which case $\left(X_{k} Z_{k}^{r_{k}}\right)^{d}=I$, where $I$ is the identity operator on the Hilbert space of one qudit. The third equality follows from the following fact: For each $s \in \mathbb{Z}_{d}$ and $l \in\{1,2, \ldots, d-1\}$, we have

$$
\begin{aligned}
& \sum_{r \in \mathbb{Z}_{d}} \omega^{-s r}\left(X Z^{r}\right)^{l}=X^{l} \sum_{r \in \mathbb{Z}_{d}} \omega^{r[l(l-1) / 2-s]} Z^{r l} \\
& =\sum_{j=0}^{d-1}|j+l\rangle\langle j|\left(\sum_{r \in \mathbb{Z}_{d}} \omega^{r[l(l-1) / 2+j l-s]}\right) .
\end{aligned}
$$

The last term in the parentheses vanishes except when $l(l-1) / 2+j l-s=0 \bmod d$, in which case it equals $d$. If $d$ is an odd prime and $l \neq 0$, then the equation $l(l-1) / 2+j l-s=0 \bmod d$ for each $s$ has a unique solution for $j \in \mathbb{Z}_{d}$, and the map from $s$ to the solution $j$ is one to one, so the third equality in Eq. (B2) holds.

To clarify why the above proof does not work when $d$ is an odd number that is not a prime, suppose $l$ is a divisor of $d$. Then the equation $l(l-1) / 2+j l-s=0 \bmod d$ has multiple solutions when $s$ is a multiple of $l$, while it has no solution otherwise, so the third equality in Eq. (B2) does not hold in this case. Therefore, we need to assume that $d$ is an odd prime in order to construct an optimal protocol based on Pauli measurements.

\section{Appendix C: Proofs of Eqs. (22) and (24)}

Proof of Eq. 22, According to Eq. 20, we have

$$
\left|\psi_{h t}\right\rangle=\frac{1}{\sqrt{d}} \sum_{j=0}^{d-1} \mathrm{e}^{\mathrm{i} \theta_{h t j}}|j\rangle=\frac{1}{\sqrt{d}} \sum_{j=0}^{d-1} \omega^{t j} \mu^{h\left(\begin{array}{c}
j \\
2
\end{array}\right)}|j\rangle .
$$

Therefore,

$$
\begin{gathered}
X W^{h} \sqrt{d}\left|\psi_{h t}\right\rangle=\sum_{j=0}^{d-2} \omega^{t j} \mu^{h\left[\left(\begin{array}{l}
j \\
2
\end{array}\right)+j\right]}|j+1\rangle \\
+\omega^{-t} \mu^{h\left[\left(\begin{array}{c}
d-1 \\
2
\end{array}\right)-(d-1)(d-2) / 2\right]}|0\rangle \\
=\omega^{-t} \sum_{j=0}^{d-1} \omega^{t j} \mu^{h\left(\begin{array}{c}
j \\
2
\end{array}\right)}|j\rangle=\omega^{-t} \sqrt{d}\left|\psi_{h t}\right\rangle .
\end{gathered}
$$

It follows that $\left|\psi_{h t}\right\rangle$ is an eigenstate of $X W^{h}$ with eigenvalue $\omega^{-t}$, which implies Eq. 22 .

Alternatively, Eq. 22 can be proved as follows.

$$
\begin{aligned}
& \sum_{t \in \mathbb{Z}_{d}} \omega^{-t}\left(\left|\psi_{h t}\right\rangle\left\langle\psi_{h t}\right|\right) \\
& =\frac{1}{d} \sum_{j, j^{\prime}=0}^{d-1}\left(\left|j^{\prime}\right\rangle\langle j|\right)\left(\mathrm{e}^{\mathrm{i} \pi h\left(j^{\prime}-j\right)\left(j^{\prime}+j-1\right) / m} \sum_{t \in \mathbb{Z}_{d}} \omega^{t\left(j^{\prime}-j-1\right)}\right) \\
& =\sum_{j=0}^{d-1}(|\hat{j}\rangle\langle j|)\left(\mathrm{e}^{\mathrm{i} \pi h(\hat{j}-j)(\hat{j}+j-1) / m}\right) \\
& =\mu^{-h(d-1)(d-2) / 2}(|0\rangle\langle d-1|)+\sum_{j=0}^{d-2} \mu^{h j}(|j+1\rangle\langle j|) \\
& =X W^{h},
\end{aligned}
$$

where $\hat{j}=j+1$ if $j \leq d-2$ and $\hat{j}=0$ if $j=d-1$.

Proof of Eq. (24). The sum of all test projectors $P_{\mathbf{h}}$ that satisfy the condition $\sum_{k} h_{k}=0 \bmod m$ can be expressed as

$$
\begin{aligned}
\sum_{\mathbf{h}} P_{\mathbf{h}} & =\frac{m^{n-1}}{d} \mathbb{1}+\frac{1}{d} \sum_{l=1}^{d-1} \sum_{\mathbf{h}} \prod_{k=1}^{n}\left(X_{k} W_{k}^{h_{k}}\right)^{l} \\
& =\frac{m^{n-1}}{d} \mathbb{1}+\frac{1}{d m} \sum_{l=1}^{d-1} \sum_{s \in \mathbb{Z}_{m}}\left[\sum_{h=1}^{m} \mu^{-s h}\left(X W^{h}\right)^{l}\right]^{\otimes n} \\
& =\frac{m^{n-1}}{d} \mathbb{1}+\frac{1}{d m} \sum_{l=1}^{d-1} \sum_{j=0}^{d-1}(m|j+l\rangle\langle j|)^{\otimes n} \\
& =\frac{m^{n-1}}{d}\left[\mathbb{1}+\sum_{j^{\prime} \neq j}\left(\left|j^{\prime}\right\rangle\langle j|\right)^{\otimes n}\right],
\end{aligned}
$$

which implies Eq. (24). To derive the third equality, for each $s \in \mathbb{Z}_{m}$ and $l=1,2, \ldots, d-1$, define

$$
f(s, l):=\sum_{h=1}^{m} \mu^{-s h}\left(X W^{h}\right)^{l}
$$

Thanks to Eq. 22, in the main text or Eq. (C3), we have

$$
\begin{aligned}
f(s, l)= & \sum_{h=1}^{m} \mu^{-s h} \sum_{t \in \mathbb{Z}_{d}} \omega^{-t l}\left|\psi_{h t}\right\rangle\left\langle\psi_{h t}\right| \\
= & \frac{1}{d} \sum_{j, j^{\prime}=0}^{d-1}\left(\left|j^{\prime}\right\rangle\langle j|\right)\left(\sum_{t \in \mathbb{Z}_{d}} \omega^{t\left(j^{\prime}-j-l\right)}\right) \\
& \times\left(\sum_{h=1}^{m} \mu^{h\left[\left(j^{\prime}-j\right)\left(j^{\prime}+j-1\right) / 2-s\right]}\right) \\
= & \sum_{j=0}^{d-1}(|\hat{j}\rangle\langle j|)\left(\sum_{h=1}^{m} \mu^{h[g(j, l, d)-s]}\right),
\end{aligned}
$$


where

$$
\begin{aligned}
\hat{j} & := \begin{cases}j+l & j+l \leq d-1, \\
j+l-d & j+l \geq d,\end{cases} \\
g(j, l, d) & :=\frac{1}{2}(\hat{j}-j)(\hat{j}+j-1) .
\end{aligned}
$$

The last term in the parentheses in Eq. C6 vanishes except when

$$
g(j, l, d)-s=0 \quad \bmod m,
$$

in which case it is equal to $m$. For given $l$ and $j$, note that Eq. (C9) has a unique solution for $s \in \mathbb{Z}_{m}$. Conversely, for each $l \in\{1,2, \ldots, d-1\}$ and $s \in \mathbb{Z}_{m}$, Eq. (C9) has at most one solution for $j \in\{0,1, \ldots, d-1\}$ by Lemma 3 below given that $m \geq\left\lceil\frac{3}{4}(d-1)^{2}\right\rceil$. This result implies the third equality in Eq. (C4) and completes the proof of Eq. 24.

Lemma 3. Let

$$
g_{m}(j, l, d):=g(j, l, d) \bmod m,
$$

where $g(j, l, d)$ is defined in Eq. (C8). Suppose $d \geq 3$, $m \geq\left\lceil\frac{3}{4}(d-1)^{2}\right\rceil$, and $l \in\{1,2, \ldots, d-1\}$; then $g_{m}(j, l, d)$ is injective in $j$ for $j \in\{0,1, \ldots, d-1\}$.

This lemma follows from Proposition 4.3 in Ref. 41. Here we present a self-contained proof for completeness.

Proof. When $j \in\{0, \ldots, d-l-1\}$, the function $g(j, l, d)$ is monotonically increasing in $j$, and we have

$$
0 \leq l(l-1) / 2 \leq g(j, l, d) \leq l(2 d-l-3) / 2<m
$$

given that $m \geq\left\lceil\frac{3}{4}(d-1)^{2}\right\rceil$. When $j \in\{d-l, \ldots, d-1\}$ by contrast, $g(j, l, d)$ is monotonically decreasing in $j$, and we have

$$
\begin{aligned}
& -m<(l-d)(d+l-3) / 2 \leq g(j, l, d) \\
& \leq(l-d)(d-l-1) / 2 \leq 0 .
\end{aligned}
$$

In addition, it is straightforward to verify that

$$
l(2 d-l-3) / 2<(l-d)(d+l-3) / 2+m .
$$

Therefore, the two sets of numbers $\left\{g_{m}(j, l, d)\right\}_{j=0}^{d-l-1}$ and $\left\{g_{m}(j, l, d)\right\}_{j=d-l}^{d-1}$ have no intersection; moreover, all the numbers $g_{m}(0, l, d), g_{m}(1, l, d), \ldots, g_{m}(d-1, l, d)$ are distinct, which confirms Lemma 3 .

\section{Appendix D: Alternative optimal protocol for verifying GHZ-like states}

In the main text we proposed an optimal strategy for verifying the GHZ-like state $|\xi\rangle=\sum_{j=0}^{d-1} \lambda_{j}|j\rangle^{\otimes n}$ based on one-way LOCC, which requires only $1+2^{n-1}$ distinct tests when $d=2$ and $1+m^{n-1}$ distinct tests with $m \geq$ $\left\lceil\frac{3}{4}(d-1)^{2}\right\rceil$ when $d \geq 3$. Here we propose an alternative optimal protocol using much fewer measurement settings, assuming that the local dimension $d$ is an odd prime. In addition, for each test, all parties except for one of them can perform Pauli measurements as in the case of qubits. The underlying idea is similar to the construction of the strategy $\Omega_{\mathrm{V}}$ in Sec. IVB

For each string $\mathbf{r} \in \mathbb{Z}_{d}^{n}$ with $\sum_{k} r_{k}=0 \bmod d$, define the test projector

$$
P_{\mathbf{r}}^{\prime}:=\left(I^{\otimes(n-1)} \otimes M\right) P_{\mathbf{r}}\left(I^{\otimes(n-1)} \otimes M\right),
$$

where $M:=\sqrt{d} \operatorname{diag}\left(\lambda_{0}, \lambda_{1}, \ldots, \lambda_{d-1}\right)$, and $P_{\mathbf{r}}$ is the test projector given in Eq. (17). Then $P_{\mathbf{r}}^{\prime}$ can be realized by adaptive local projective measurements as described in Sec. IV B. According to Eq. (B2), we have

$$
\frac{1}{d^{n-1}} \sum_{\mathbf{r}} P_{\mathbf{r}}=\frac{1}{d}\left[\mathbb{1}+\sum_{j \neq j^{\prime}}\left(\left|j^{\prime}\right\rangle\langle j|\right)^{\otimes n}\right] .
$$

As a corollary,

$$
\begin{aligned}
& \frac{1}{d^{n-1}} \sum_{\mathbf{r}} P_{\mathbf{r}}^{\prime}=\left(I^{\otimes(n-1)} \otimes M\right)\left(\frac{\sum_{\mathbf{r}} P_{\mathbf{r}}}{d^{n-1}}\right)\left(I^{\otimes(n-1)} \otimes M\right) \\
& =|\xi\rangle\langle\xi|+I^{\otimes(n-1)} \otimes \rho_{n}-\sum_{j=0}^{d-1} \lambda_{j}^{2}(|j\rangle\langle j|)^{\otimes n}, \quad \quad \text { (D3) }
\end{aligned}
$$

where $\rho_{n}=\sum_{j=0}^{d-1} \lambda_{j}^{2}|j\rangle\langle j|$ is the reduced state for party $n$. Note that the right-hand side in Eq. (D3) is identical to its counterpart in Eq. (38).

Suppose we perform the test $P_{0}$ with probability $p$ and the other tests $P_{\mathbf{r}}^{\prime}$ with probability $(1-p) / d^{n-1}$ each. Then the verification operator reads

$$
\Omega_{\mathrm{V}}^{\prime}=p P_{0}+\frac{1-p}{d^{n-1}} \sum_{\mathbf{r}} P_{\mathbf{r}}^{\prime}
$$

and we have

$$
\beta\left(\Omega_{\mathrm{V}}^{\prime}\right)=\beta\left(\Omega_{\mathrm{V}}\right)=\max \left\{p,(1-p) \lambda_{0}^{2}\right\} \geq \frac{\lambda_{0}^{2}}{1+\lambda_{0}^{2}}
$$

as in Eq. (39) in the main text. The lower bound is attained when $p=\lambda_{0}^{2} /\left(1+\lambda_{0}^{2}\right)$, in which case we can achieve the maximum spectral gap $\nu\left(\Omega_{\mathrm{V}}^{\prime}\right)=1 /\left(1+\lambda_{0}^{2}\right)$ as in Eq. 40. When $d$ is an odd prime, therefore, $1+d^{n-1}$ distinct tests are sufficient for constructing a strategy that is equivalent to $\Omega_{\mathrm{V}}$ in Sec. IVB, which is optimal among all strategies based on one-way LOCC. Nevertheless, the efficiency can be improved further by virtue of more communications as employed in the construction of $\Omega_{\mathrm{VI}}$ in Sec. IVC. 
[1] D. M. Greenberger, M. A. Horne, and A. Zeilinger, in: Bell's Theorem, Quantum Theory, and Conceptions of the universe, edited by M. Kafatos (Kluwer Academic, Dordrecht, 1989), pp. 69-72.

[2] D. M. Greenberger, M. A. Horne, A. Shimony, and A. Zeilinger, Bell's theorem without inequalities. Am. J. Phys. 58, 1131 (1990).

[3] O. Gühne and G. Tóth, Entanglement detection. Phys. Rep. 474, 1 (2009).

[4] W. Tittel, H. Zbinden, and N. Gisin, Experimental demonstration of quantum secret sharing. Phys. Rev. A 63, 042301 (2001).

[5] Y.-A. Chen et al. Experimental quantum secret sharing and third-man quantum cryptography. Phys. Rev. Lett. 95, 200502 (2005).

[6] Y. W. Cheong, S.-W. Lee, J. Lee, and H.-W. Lee, Entanglement purification for high-dimensional multipartite systems. Phys. Rev. A 76, 042314 (2007).

[7] Z. Zhao, Y.-A. Chen, A.-N. Zhang, T. Yang, H. J. Briegel, and J.-W. Pan, Experimental demonstration of five-photon entanglement and open-destination teleportation. Nature 430, 54 (2004).

[8] W. McCutcheon et al. Experimental verification of multipartite entanglement in quantum networks. Nat. Commun. 7, 13251 (2016).

[9] M. Hayashi and T. Koshiba, Secure Modulo ZeroSum Randomness as Cryptographic Resource. (2018), https://eprint.iacr.org/2018/802.

[10] V. Scarani and N. Gisin, Spectral decomposition of Bell's operators for qubits. J. Phys. A: Math. Gen. 34, 6043 (2001).

[11] C. Zhang, Y.-F. Huang, Z. Wang, B.-H. Liu, C.-F. Li, and G.-C. Guo, Experimental Greenberger-Horne-ZeilingerType Six-Photon Quantum Nonlocality. Phys. Rev. Lett. 115, 260402 (2015).

[12] K. J. Resch, P. Walther, and A. Zeilinger, Full Characterization of a Three-Photon Greenberger-Horne-Zeilinger State Using Quantum State Tomography. Phys. Rev. Lett. 94, 070402 (2005).

[13] X.-L. Wang et al. Experimental ten-photon entanglement. Phys. Rev. Lett. 117, 210502 (2016).

[14] C. Song et al. 10-Qubit Entanglement and Parallel Logic Operations with a Superconducting Circuit. Phys. Rev. Lett. 119, 180511 (2017).

[15] M. Erhard, M. Malik, M. Krenn, and A. Zeilinger, Experimental Greenberger-Horne-Zeilinger entanglement beyond qubits. Nat. Photon. 12, 759 (2018).

[16] Y. Ji, J. Bian, X. Chen, J. Li, X. Nie, H. Zhou, and X. Peng, Experimental preparation of Greenberger-HorneZeilinger states in an Ising spin model by partially suppressing the nonadiabatic transitions. Phys. Rev. A 99, 032323 (2019).

[17] D. Cruz et al. Efficient quantum algorithms for $G H Z$ and $W$ states, and implementation on the IBM quantum computer. Adv. Quantum Technol. 2, 1900015 (2019).

[18] P. Imany et al. High-dimensional optical quantum logic in large operational spaces. npj Quantum Inf. 5, 59 (2019).

[19] H. Häffner et al. Scalable multiparticle entanglement of trapped ions. Nature 438, 643 (2005).

[20] S. T. Flammia and Y.-K. Liu, Direct Fidelity Estimation from Few Pauli Measurements. Phys. Rev. Lett. 106,
230501 (2011)

[21] M. Hayashi, K. Matsumoto, and Y. Tsuda, A study of LOCC-detection of a maximally entangled state using hypothesis testing. J. Phys. A: Math. Gen. 39, 14427 (2006).

[22] L. Aolita, C. Gogolin, M. Kliesch, and J. Eisert, Reliable quantum certification of photonic state preparations. Nat. Commun. 6, 8498 (2015).

[23] D. Hangleiter, M. Kliesch, M. Schwarz, and J. Eisert, Direct certification of a class of quantum simulations. Quantum Sci. Technol. 2, 015004 (2017).

[24] S. Pallister, N. Linden, and A. Montanaro, Optimal Verification of Entangled States with Local Measurements. Phys. Rev. Lett. 120, 170502 (2018).

[25] H. Zhu and M. Hayashi, Efficient Verification of Pure Quantum States in the Adversarial Scenario. Phys. Rev. Lett. 123, 260504 (2019).

[26] H. Zhu and M. Hayashi, General framework for verifying pure quantum states in the adversarial scenario. Phys. Rev. A 100, 062335 (2019).

[27] M. Hayashi and T. Morimae, Verifiable MeasurementOnly Blind Quantum Computing with Stabilizer Testing. Phys. Rev. Lett. 115, 220502 (2015).

[28] A. Kalev, A. Kyrillidis, and N. M. Linke, Validating and certifying stabilizer states. Phys. Rev. A 99, 042337 (2019).

[29] H. Zhu and M. Hayashi, Efficient Verification of Hypergraph States. Phys. Rev. Appl. 12, 054047 (2019).

[30] Y.-C. Liu, X.-D. Yu, J. Shang, H. Zhu, and X. Zhang, Efficient Verification of Dicke States. Phys. Rev. Appl. 12, 044020 (2019).

[31] M. Hayashi, Group theoretical study of LOCC-detection of maximally entangled states using hypothesis testing. New J. Phys. 11, 043028 (2009).

[32] H. Zhu and M. Hayashi, Optimal verification and fidelity estimation of maximally entangled states. Phys. Rev. A 99, 052346 (2019).

[33] Z. Li, Y.-G. Han, and H. Zhu, Efficient verification of bipartite pure states. Phys. Rev. A 100, 032316 (2019).

[34] K. Wang and M. Hayashi, Optimal verification of twoqubit pure states. Phys. Rev. A 100, 032315 (2019).

[35] X.-D. Yu, J. Shang, and O. Gühne, Optimal verification of general bipartite pure states. npj Quantum Inf. 5, 112 (2019).

[36] H. Zhu and H. Zhang, Efficient verification of quantum gates with local operations. Phys. Rev. A 101, 042316 (2020).

[37] Y.-C. Liu, J. Shang, X.-D. Yu, and X. Zhang, Efficient and practical verification of quantum processes. Phys. Rev. A 101, 042315 (2020).

[38] P. Zeng, Y. Zhou, and Z. Liu, Quantum gate verification and its application in property testing. (2019), arXiv:1911.06855.

[39] J. M. Renes, R. Blume-Kohout, A. J. Scott, and C. M. Caves, Symmetric informationally complete quantum measurements. J. Math. Phys. 45, 2171 (2004).

[40] A. J. Scott, Tight informationally complete quantum measurements. J. Phys. A: Math. Gen. 39, 13507 (2006).

[41] A. Roy and A. J. Scott, Weighted complex projective 2-designs from bases: Optimal state determination by orthogonal measurements. J. Math. Phys. 48, 072110 
(2007).

[42] G. Tóth and O. Gühne, Detecting Genuine Multipartite Entanglement with Two Local Measurements. Phys. Rev. Lett. 94, 060501 (2005).

[43] Y. Zhou, Entanglement detection under coherent noise: Greenberger-Horne-Zeilinger-like states. Phys. Rev. A 101, 012301 (2020).

[44] A. Dimić and B. Dakić, Single-copy entanglement detection. npj Quantum Inf. 4, 11 (2018).

[45] G. Gordon and G. Rigolin, Generalized quantum-state sharing. Phys. Rev. A 73, 062316 (2006).

[46] M. Żukowski, Č. Brukner, W. Laskowski, and M. Wieśniak, Do All Pure Entangled States Violate Bell's Inequalities for Correlation Functions? Phys. Rev. Lett. 88, 210402 (2002).

[47] J. L. Cereceda, Hardy's nonlocality for generalized $n$ particle GHZ states. Phys. Lett. A 327, 433 (2004).

[48] D. Leibfried et al. Toward Heisenberg-Limited Spec- troscopy with Multiparticle Entangled States. Science 304, 1476 (2004).

[49] L. Jiang et al. Repetitive Readout of a Single Electronic Spin via Quantum Logic with Nuclear Spin Ancillae. Science 326, 267 (2009).

[50] I. D. Ivanović, Geometrical description of quantal state determination. J. Phys. A: Math. Gen. 14, 3241 (1981).

[51] W. K. Wootters and B. D. Fields, Optimal statedetermination by mutually unbiased measurements. Ann. Phys. 191, 363 (1989).

[52] T. Durt, B.-G. Englert, I. Bengtsson, and K. Życzkowski, On mutually unbiased bases. Int. J. Quantum Inf. 08, 535 (2010).

[53] Y. Takeuchi and T. Morimae, Verification of Many-Qubit States. Phys. Rev. X 8, 021060 (2018).

[54] D. Mayers and A. Yao, Self testing quantum apparatus. Quantum Inf. Comput. 4, 273-286 (2004).

[55] I. Šupić and J. Bowles, Self-testing of quantum systems: a review. (2019), arXiv:1904.10042. 